has led Stewart and Lee (Planta, 120, $279 ; 1974)$ to investigate the possibility that proline may be associated with the tolerance of physiological drought in halophytes. They analysed the free amino acid content of a number of halophytes from salt marshes in western Britain and found that in many (for example Aster tripolium, Armeria maritima, Puccinellia maritima and Triglochin maritima) the concentration of proline was higher than that of any other single amino acid, often by a factor of ten. In some species proline represented $70 \%$ of the free amino acids and in most it exceeded $30 \%$. But Plantago maritima had a low proline content; this exception demonstrates that proline accumulation is not a universal feature of halophytes.

Using Triglochin maritima Stewart and Lee were able to show that proline build-up is enhanced by high salinity in the surroundings, just as Waldren and Teare had demonstrated in the case of water stress. They also found that different populations of certain species, which were geographically isolated and which had experienced different salinities over long periods of time, had varying capacities for the accumulation of proline. For example, Armeria maritima, the thrift, is a species which occurs both as a maritime halophyte and as a species of arctic-alpine habitats on many British mountains. Palaeoecological studies suggest that the species was widespread in distribution during the cold and disturbed conditions at the close of the last glaciation, some 10,000 years ago. Populations have subsequently been isolated on mountain tops and coastal habitats as forest has spread and eliminated this shade-intolerant species from many habitats. When tested under a variety of salinities, Armeria populations from inland situations showed only a limited capacity for proline accumulation. The response of coastal Armeria samples was about three times as great as those from inland. Such a difference in response is reminiscent of the varietal adaptation to proline accumulation as a response to drought found in barley (Singh et al.; 1972).

It is known that the enzymes of some halophytes are not able to operate in media of high salt concentration (for example, the work of Flowers, J. exp. Bot., 23, 310; 1972, on Sueda maritima). Sodium chloride within halophytes must, therefore, be physically separated from the cellular enzyme systems, presumably by storage in the vacuole. Stewart and Lee have examined the effect of elevated proline levels upon the activity of several enzymes in Triglochin maritima; they found no depression of activity. They suggest, therefore, that proline could provide the means of cytoplasmic osmotic adjustment in those halophytes where proline accumulation has been observed. Perhaps it serves a similar function in drought-stressed tissues where osmotic compensation may be required within the cytoplasm of affected cells. Apart from the elucidation of the precise role of proline in these processes, one of the most interesting developments in this work is likely to emerge from the exceptions to the general rules-exceptions like Plantago maritima.

\section{Laser studies of atomic and molecular collisions}

\section{from G. Duxbury}

IN the last fifteen years there has been an increasing interest in the experimental and theoretical study of the energy transfer processes occurring in atomic and molecular collisions. One of the main experimental techniques which has been used is that of crossed molecular beams, which requires very expensive ultra-high vacuum apparatus. An alternative way of obtaining the information is based upon experiments using single frequency narrow linewidth lasers. In these experiments use is made of the velocity selective interaction of monochromatic radiation with gaseous atoms and molecules. The information obtained from the laser experiments is similar to that from the crossed beam experiments, but does not require ultrahigh vacuum techniques for its success. In addition, information can be gained, not only on the changes of translational energy, but also on changes in rotational energy. As examples of the types of experiment that can be carried out, two different ones are described, one of which has been carried out and one which has recently been proposed.

If a single-frequency laser emits radiation of frequency $\nu$ which differs slightly from the resonance frequency $\nu_{0}$ of the atoms or molecules which absorb the radiation, the Doppler shift will make the laser radiation appear exactly on resonance only for those molecules whose component of velocity along the laser beam is $v=c\left(v-v_{0}\right) / v_{0}$, where $c$ is the velocity of light. If the molecule irradiated is allowed to collide with a heavy target, the velocity dependence of the collision cross section for the production of a particular excited state can be determined by measuring the ratio of the fluorescence from this state, to that of the laser excited resonance fluorescence as the laser frequency is changed. Phillips and Pritchard (Phys. Rev. Lett., 33, 1254 ; 1974) have proposed that this technique should give velocity resolutions comparable with that obtained using the crossed molecular beam method, providing that the target is at least five to ten times heavier than the atom or molecule excited. They also suggest that since the velocity distribution in the laser experiment is known exactly, the true cross section can be deconvoluted from the measured one very accurately, so that this compensates for the slightly poorer velocity resolution of the laser experiment. It is proposed that, in some circumstances, state selection can be achieved by the use of a polarised laser beam. This should give a preferential orientation of either the orbital angular momentum vector, or of an electric dipole moment, along the direction of propagation of the radiation. The experiments described above are for the linear absorption region.

An alternative method of studying energy transfer that has already been demonstrated utilises saturation effects produced in low pressure gases by high power lasers. Under these conditions 'holes' are produced in the population of energy levels associated with the absorbing transition. Good examples of this type of behaviour are provided by the elegant double resonance experiments carried out by Freund, Johns, McKellar and Oka (J. chem. Phys., 59. $3445 ; 1973)$. They studied two, three and four level systems in the vibration rotation spectrum of ammonia. The main effects that they demonstrated were the monitoring of a hole in the population of a vibration-rotation energy level caused by a strong twophoton pumping source, by a weak two-photon signal source, and the observation that holes burnt in the population of one level could be transferred to other levels by collisions. Twophoton pumping is not an essential requisite, and can be replaced by tunable laser sources once these become available. By fine tuning both the pumping and the monitoring sources different velocity molecules were studied, and by larger variations of the monitoring source energy transfer to different rotational energy levels was studied. Thus from one type of experiment it is possible to obtain information about both the magnitude of the intermolecular interaction $V(r)$ from the translational changes, and the angular dependence of the intermolecular interaction $V(\theta, \varphi)$ from the rotational changes.

These sorts of study are only possible when the predominant cause of spectral line broadening is the Doppler effect, as in the infrared and visible regions. In the microwave region the lines are predominantly pressure broadened and double resonance then gives information only on rotational state changes, but not on changes in translational energy. 